\title{
Composite Sinusoidal Nanograting With Long-Range SERS Effect for Label-Free TNT Detection
}

\author{
Cheng XIAO*, Zhibin CHEN, Mengze QIN, Dongxiao ZHANG, and Lei FAN \\ Department of Electronic and Optical Engineering, Shijiazhuang Campus of Army Engineering University, Shijiazhuang \\ 050003, China \\ *Corresponding author: Cheng XIAO \\ E-mail: xc_nanking@163.com
}

\begin{abstract}
A composite one-dimensional (1D) Ag sinusoidal nanograting aiming at label-free surface enhanced Raman scattering (SERS) detection of TNT with robust and reproducible enhancements is discussed. 1D periodic sinusoidal $\mathrm{SiO}_{2}$ grating followed by $\mathrm{Ag}$ evaporation is proposed for the creation of reproducible and effective SERS substrate based on surface plasmon polaritons (SPPs). The optimal structure of 1D sinusoidal nanograting and its long-range SERS effect are analyzed by using the finite difference time domain (FDTD). Simulation SERS enhancement factor (EF) can be 5 orders of magnitude as possible. This SERS substrate is prepared by the interference photolithography technology, its SERS performance is tested by Rh6G detection experiments, and the actual test EF is about $10^{4}$. The label-free SERS detection capacity of TNT is demonstrated in the experiment.
\end{abstract}

Keywords: Sinusoidal grating; surface plasmon polaritons; long-range SERS effect; label-free

Citation: Cheng XIAO, Zhibin CHEN, Mengze QIN, Dongxiao ZHANG, and Lei FAN, "Composite Sinusoidal Nanograting With Long-Range SERS Effect for Label-Free TNT Detection,” Photonic Sensors, 2018, 8(3): 278-288.

\section{Introduction}

At present, the main surface enhanced Raman scattering (SERS) substrates integrated with microfluidics for trace explosives detection are rough noble metallic substrates, self-assembly noble metallic nanoparticles substrates, noble metallic periodic arrays, and composite graphene substrates [1]. Due to the surface irregularity of the noble metallic substrate, its reproducibility is poor. Uncontrolled nanoparticles aggregation of self-assembly noble metallic nanoparticles substrate also leads to poor reproducibility of the SERS signal and seriously limits its wide applications [2]. Arrays nanostructures are manufactured by nanofabrication techniques, which provide enhancements with good repeatability, but are rather expensive and time consuming to be fabricated [3-5]. Besides, the hot spots areas of these nanostructures SERS substrates, such as rough surface, nanospheres, nanopillar [6], nanotriangles [7], nanowires [8], and composite graphene substrate [9], are relatively small, and only a short-range SERS effect exists in these substrates [10]. So detected explosives molecules should be absorbed on these nanostructures with label reagents, and nanostructures need to be cleaned before the next application [11], thus it cannot be used in the real-time and label-free SERS detection of trace explosives.

In this work, a potential reliable SERS substrate

Received: 11March 2018 / Revised: 28 May 2018

(C) The Author(s) 2018.This article is published with open access at Springerlink.com

DOI: $10.1007 / \mathrm{s} 13320-018-0497-6$

Article type: Regular 
based on one-dimensional (1D) sinusoidal $\mathrm{SiO}_{2}$ nanograting followed by $\mathrm{Ag}$ evaporation is offered. A large-area and low-cost SERS substrate can be achieved simply and rapidly by this method. From theoretical and simulation points of view, the excitation surface plasmon polaritons (SPPs) performance of this substrate is researched, and it has a long-range SERS effect. So it has potential in label-free testing of trace TNT. In the experiments, composite 1D sinusoidal Ag nanogratings are fabricated, and their label-free SERS testing performance of trace TNT is studied.

\section{Theoretical analysis of SPPs-active}

Based on the dielectric functions of metals [12], we investigate why the propagating SPPs can exist at the dielectric-metal interfaces. In addition, we derive the dispersion relation of this surface wave and discuss its unique properties.

As shown in Fig. 1, the propagating SPPs represent longitudinal oscillations of the surface charges. Hence, we assume the wave propagates along the $x$ axis, which shows no spatial variation along the $y$ axis. The electric field is therefore in the $x-z$ plane, and the magnetic field is along the $y$ axis. The amplitudes of the $x$ - and $z$-components of the electric field in half spaces 1 and 2 are denoted as $E_{x_{1}}, E_{z_{1}}, E_{x_{2}}$ and $E_{z_{2}}$, respectively. The amplitudes of the magnetic fields in half spaces 1 and 2 are denoted as $H_{y_{1}}$ and $H_{y_{2}}$, respectively. We therefore have the following relationships:

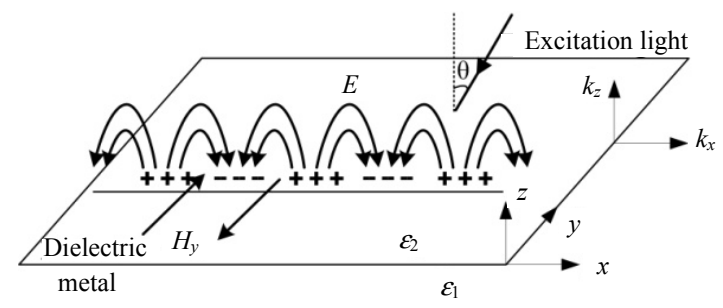

Fig. 1 Schematic illustration of SPPs at a metal-dielectric interface.

$$
\begin{gathered}
\mathbf{E}_{1}=\left(\hat{x} E_{x_{1}}+\hat{z} E_{z_{1}}\right) \exp \left[\mathrm{i}\left(k_{x_{1}} x-\omega t\right)\right] \exp \left(\mathrm{i} k_{z_{1}} z\right) \\
\mathbf{H}_{1}=\hat{y} H_{y_{1}} \exp \left[\mathrm{i}\left(k_{x_{1}} x-\omega t\right)\right] \exp \left(\mathrm{i} k_{z_{1}} z\right)
\end{gathered}
$$

$$
\begin{gathered}
\mathbf{E}_{2}=\left(\hat{x} E_{x_{2}}+\hat{z} E_{z_{2}}\right) \exp \left[\mathrm{i}\left(k_{x_{2}} x-\omega t\right)\right] \exp \left(\mathrm{i} k_{z_{2}} z\right) \\
\mathbf{H}_{2}=\hat{y} H_{y_{2}} \exp \left[\mathrm{i}\left(k_{x_{2}} x-\omega t\right)\right] \exp \left(\mathrm{i} k_{z_{2}} z\right) .
\end{gathered}
$$

From the requirement of Maxwell's equations that the tangential components of the electric and magnetic fields are continuous at the boundary, we obtain $k_{x_{1}}=k_{x_{2}}=k_{x}, \quad E_{x_{1}}=E_{x_{2}}=E_{x}, \quad$ and $H_{y_{1}}=H_{y_{2}}$. Considering that the displacement fields in both half spaces are source free, i.e. $\nabla \cdot \mathbf{D}=0$, we obtain $k_{x} E_{x}+k_{z_{j}} E_{z_{j}}=0$. From Maxwell's equations, we have the following relationships:

$$
\begin{aligned}
& \nabla \times \mathbf{E}=-\mu_{0} \frac{\partial \mathbf{H}}{\partial t} \\
& \nabla^{2} \mathbf{E}+\frac{\omega^{2}}{c^{2}} \varepsilon \mathbf{E}=0 .
\end{aligned}
$$

And based on the above equations, we have

$$
\begin{gathered}
H_{y_{j}}=\omega \varepsilon_{j} \varepsilon_{0} \frac{E_{x}}{k_{z_{j}}}, j=1,2 \\
\omega^{2} \mu_{0} \varepsilon_{0} \varepsilon_{j}=k_{x}^{2}+k_{z_{j}}^{2}, j=1,2 .
\end{gathered}
$$

Combining $H_{y_{1}}=H_{y_{2}}$ with (7), we can obtain as follows:

$$
\frac{\varepsilon_{1}}{k_{z_{1}}}=\frac{\varepsilon_{2}}{k_{z_{2}}}
$$

We are looking for a solution bound to the interface, which requires $\mathrm{i} k_{z_{1}}>0$ and $\mathrm{i} k_{z_{2}}<0$. Then combining (8) and (9), we obtain the dispersion relationship for SPPs as follows:

$$
k_{x}=\frac{\omega}{c} \sqrt{\frac{\varepsilon_{1} \varepsilon_{2}}{\varepsilon_{1}+\varepsilon_{2}}} .
$$

The grating is a mean to couple to SPPs, as shown in Fig. 1. In order to excite SPPs, one must overcome the momentum mismatch between the excitation light propagating within a dielectric medium and SPPs propagating at the interface of the metal and dielectric [12]. As shown in Fig. 2, diffraction gratings with a periodicity $P$ can provide wave vector components in the plane of the surface with a magnitude $K=2 \pi n / P, n$ being an integer [13, 14]. For a fixed $P$, a properly chosen excitation light wavelength $\lambda_{0}[15]$ is 


$$
\lambda_{0} \approx \frac{P}{n} \sqrt{\frac{\varepsilon_{\text {metal }}\left(\lambda_{0}\right) \varepsilon_{\text {dielectric }}}{\varepsilon_{\text {metal }}\left(\lambda_{0}\right)+\varepsilon_{\text {dielectric }}}}
$$

where $\varepsilon_{\text {metal }}$ and $\varepsilon_{\text {dielectric }}$ are the dielectric constants of the metal layer and above dielectric, respectively. $\lambda_{0}$ can excite SPPs with $k_{\mathrm{spp}}$ [16]:

$$
k_{\text {spp }}=\left|k_{0} \sin \theta \pm n \frac{2 \pi}{P}\right|
$$

where $\theta$ is the incident angle of the excitation light, as shown in Fig. 1.

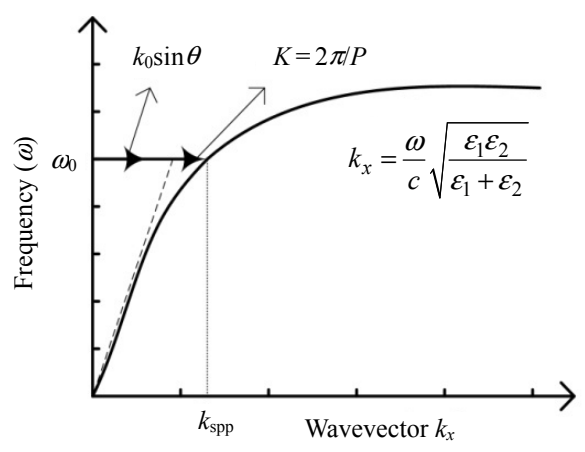

Fig. 2 Dispersion curve of SPPs.

\section{Original simulation model preparation with FDTD}

In this section, we establish the original model of 1D sinusoidal $\mathrm{Ag} / \mathrm{SiO}_{2}$ nanograting with $785-\mathrm{nm}$ excitation light. This wavelength is extensively adopted in the SERS detection. In order to choose an appropriate grating period, we carry out calculations to treat the metal as Ag with a Drude plus two-pole Lorentzian form for its dielectric constant [17]:

$$
\varepsilon_{\text {metal }}(\omega)=\varepsilon_{\infty}-\frac{\omega_{p}^{2}}{\omega^{2}+\mathrm{i} \gamma \omega}-\sum_{m=1}^{2} \frac{g_{m} \omega_{m}^{2} \Delta \varepsilon}{\omega^{2}-\omega_{m}^{2}+\mathrm{i} 2 \gamma_{m} \omega}
$$

where for $\mathrm{Ag}, \varepsilon_{\infty}=2.3646, \omega_{p}=8.7377 \mathrm{eV}$, $\gamma=0.07489 \mathrm{eV}, \quad \Delta \varepsilon=1.1831, \quad g_{1}=0.2663$, $\omega_{1}=4.3802 \mathrm{eV} \quad, \quad \gamma=0.28 \mathrm{eV} \quad, \quad \mathrm{g}_{2}=0.7337$, $\omega_{2}=5.183 \mathrm{eV}$, and $\gamma_{2}=0.5482 \mathrm{eV}$.

Substituting of (13) into (11), for this 1D sinusoidal nanograting will be integrated with microchannel, $\varepsilon_{\text {dielectric }}=1.77$, when $n=1$, we can obtain that the grating period is $570 \mathrm{~nm}$, as shown in
Fig. 3. It is obviously that the grating period is proportional to the wavelength of excitation light in the SPPs condition.

For further studying the SERS characteristic of sinusoidal $\mathrm{Ag} / \mathrm{SiO}_{2}$ nanograting, the finite difference time domain (FDTD) solutions are used to simulate the consequent SPPs phenomenon generated with the plane wave excitation. We model the electromagnetic response of the structures as shown in Fig. 4(a). The electric $\mathbf{E}(x, y, z, t)$ and magnetic $\mathbf{H}(x, y, z, t)$ fields are represented on discrete and staggered grids, and propagated in time using a leap-frog algorithm [18].

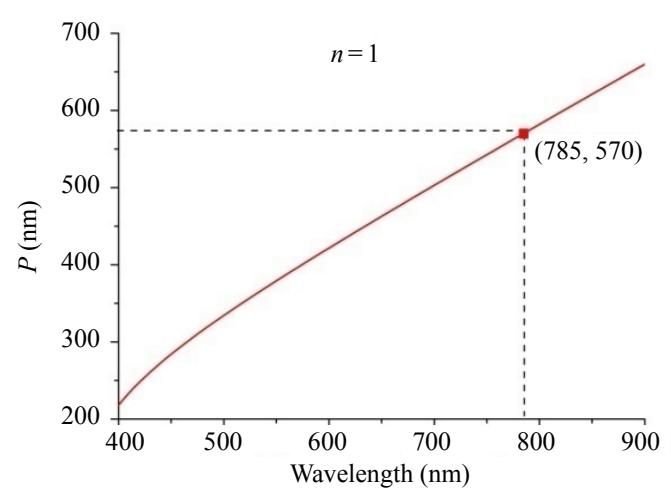

Fig. 3 Grating period vs wavelength of excitation light in SPPs condition.

Referring to the coordinate systems in Fig.4(a), the structure model consists of four layers (water, $\mathrm{Ag}$, indurated photoresist, and $\mathrm{SiO}_{2}$ ), with the period $P$, thickness $d$ of Ag layer, and amplitude $A$ of the sinusoidal grating. The medium above the Ag layer is water. Periodic boundary conditions in $x$ and $y$ axes are imposed on unit cells consistent with each structure to simulate the gratings. Perfectly matched layers (PMLs) are used to absorb field components at the grid edges in the $z$ axis [18]. The excitation light is an $x$-linearly polarized plane wave normal to the Ag layer surface injected using the plane wave approach. A frequency-domain field and power monitor is placed at the cross profile of the structure to record the near field intensity. In order to calculate the transmittance and reflectance of the sinusoidal Ag layer, further two frequency-domain 
field and power monitors are placed above the sliver layer and in the $\mathrm{SiO}_{2}$ layer, respectively. Relatively, fine grid spacing is set with $2 \mathrm{~nm}$, and simulation time with $300 \mathrm{fs}$ is applied for well converged results.

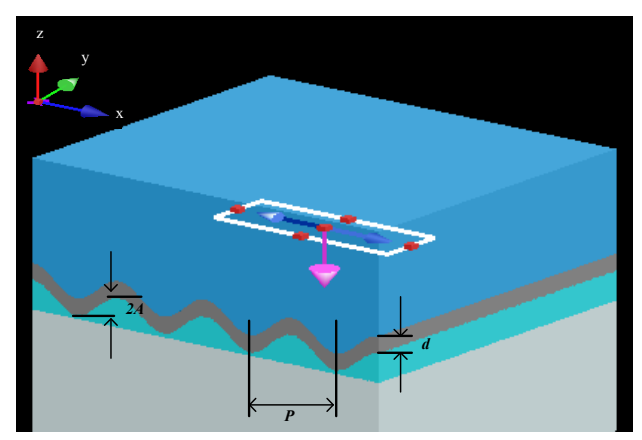

(a)

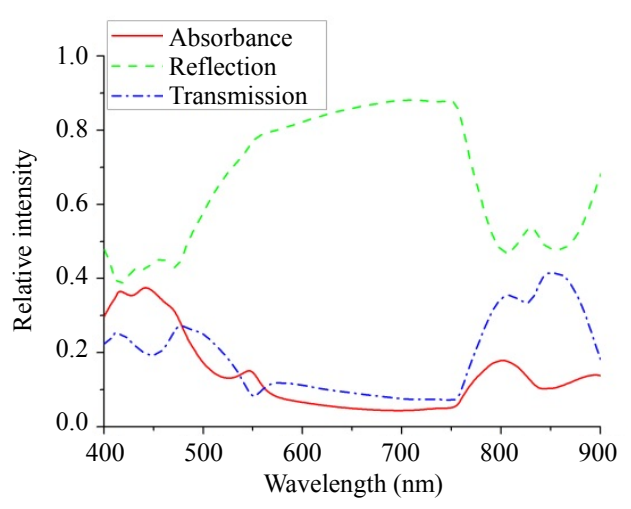

(b)

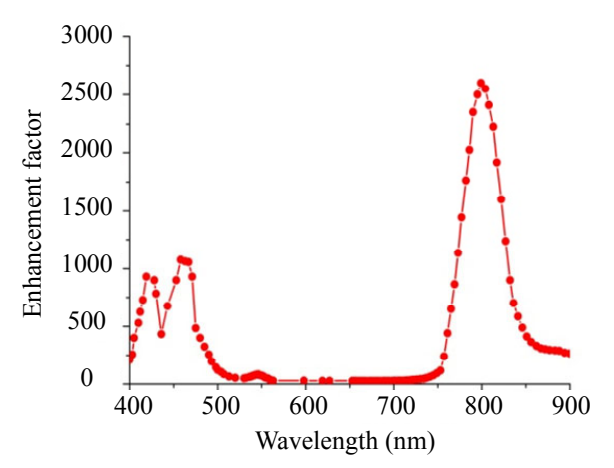

(c)

Fig. 4 Original model of the 1D sinusoidal nanograting: (a) SERS substrate based on the 1D sinusoidal Ag grating, (b) calculated absorbance, transmission, and reflection spectra of the sinusoidal Ag grating with the parameters: $A=50 \mathrm{~nm}, P=$ $570 \mathrm{~nm}$, and $d=40 \mathrm{~nm}$, and (c) simulated EF vs wavelength of excitation light.

In Fig. 4(b), the absorbance, transmittance, and reflectance of the sinusoidal $\mathrm{Ag} / \mathrm{SiO}_{2}$ grating are plotted as functions of the excitation light wavelength, indicating that in this structure one of the plasmon absorbance peaks exists when the wavelength is $802 \mathrm{~nm}$, near the desired $785 \mathrm{~nm}$. Besides, another absorbance peak exists when the wavelength is $442 \mathrm{~nm}$ and corresponds to another SPPs excitation wavelength $451 \mathrm{~nm}(n=2)$. The corresponding wavelength of the maximum SERS enhancement factor (EF) is the same as the absorbance peak wavelength, as shown in Fig.4(c). $E F \approx\left|E(\omega) / E_{0}(\omega)\right|^{4}$, where $E_{0}(\omega)$ is the electric field of the incident light, and $E(\omega)$ is the electric field near the surface of the grating. So if we want to realize more effective photon plasmon energy transfer and light focus, the excitation wavelength must be close to the maximum of the plasmon-related absorbance peak.

\section{Numerical simulation results and analysis}

The 1D periodical sinusoidal grating structure is illuminated by perpendicularly incident light for two wavelengths $532 \mathrm{~nm}$ and $785 \mathrm{~nm}$, which are typical for the existing Raman spectrophotometer and other relevant devices [19]. More structure parameters, such as the period $P$, amplitude $A$ of the sinusoidal grating and thickness $d$ of the Ag layer, and detailed excitation light parameters, such as the wavelength $\lambda$, polarization state, and polarization angle, are discussed.

\subsection{Simulation SERS EF of the 1D sinusoidal grating}

The amplitude setting of the sinusoidal grating is $50 \mathrm{~nm}$. EF is evaluated for the range of periods and $\mathrm{Ag}$ thicknesses. For such analysis, the available thicknesses of the continuous Ag film (from $20 \mathrm{~nm}$ to $200 \mathrm{~nm}$ ) and grating period (from $300 \mathrm{~nm}$ to 1140 $\mathrm{nm})$ are taken into account. Among these periods, $363 \mathrm{~nm}$ and $726 \mathrm{~nm}$ correspond to $\lambda=532 \mathrm{~nm}$, and $570 \mathrm{~nm}$ and $1140 \mathrm{~nm}$ correspond to $\lambda=785 \mathrm{~nm}$ when 
$n=1,2$, respectively.

In the case of the excitation wavelength $532 \mathrm{~nm}$, the optimal period is $363 \mathrm{~nm}$, and the Ag thickness should be near $100 \mathrm{~nm}$. For SPPs excitations with the wavelength $785 \mathrm{~nm}$, the optimal period is $570 \mathrm{~nm}$, and the $\mathrm{Ag}$ thickness is also about $100 \mathrm{~nm}$. For different period gratings, the corresponding Ag layer thickness of the maximum EF is alike. In the case where the plasmon absorbance is not efficiently excited, EF is also small. EF clearly indicates a very good agreement between the calculated absorbance of the excitation wavelength and effectivity of the SERS response. Some disagreement occurs for thinner Ag films, where the continuous film is not formed. Furthermore, some disagreement also occurs for bigger period gratings. As the period increases, the $\mathrm{Ag}$ layer tends to the flat $\mathrm{Ag}$ substrate.

In general, we can see that EF mostly depends on structure parameters. In the case of effective SPPs excitation, the strongest SERS response is observed. In Table 1, we can see that by controlling the period and metal thickness, EF can be increased to 3 orders of magnitude. Consequently, for effective pumping of excitation light energy into SPPs and optimal SERS response, an interplay of the grating period, noble metal thickness, and excitation wavelength should be taken into account.

\subsection{Period of the 1D sinusoidal grating}

The calculated absorbances as the function of the excitation light wavelength for different $\mathrm{Ag}$ layer thicknesses are plotted in Fig. 5. It is obviously that two relative strong absorbance regions exist except for $P=363 \mathrm{~nm}$. As shown in Figs. 5(b), 5(c), and 5(d), in strong absorbance regions, the absorbance remains about the same when the thickness of the Ag layer is greater than $50 \mathrm{~nm}$. So we choose an appropriate thickness $d=100 \mathrm{~nm}$ in the next simulation project.
Table $1 \mathrm{EF}$ at two excitation wavelengths as a function of the Ag layer thickness and grating period.

\begin{tabular}{|c|c|c|c|c|c|c|c|c|c|}
\hline \multirow{2}{*}{$\lambda(\mathrm{nm})$} & \multirow{2}{*}{$d(\mathrm{~nm})$} & \multicolumn{8}{|c|}{$P(\mathrm{~nm})$} \\
\hline & & 300 & 363 & 450 & 570 & 650 & 726 & 1000 & 1140 \\
\hline \multirow{8}{*}{532} & 20 & 71 & 488 & 23 & 46 & 39 & 13 & 16 & 19 \\
\hline & 40 & 81 & 983 & 28 & 53 & 209 & 28 & 37 & 39 \\
\hline & 80 & 283 & 1575 & 33 & 53 & 231 & 342 & 45 & 46 \\
\hline & 100 & 283 & 1678 & 33 & 53 & 256 & 448 & 46 & 46 \\
\hline & 120 & 375 & 1678 & 39 & 46 & 256 & 448 & 46 & 46 \\
\hline & 160 & 256 & 1575 & 39 & 46 & 256 & 410 & 46 & 46 \\
\hline & 180 & 256 & 1478 & 39 & 46 & 256 & 410 & 39 & 46 \\
\hline & 200 & 256 & 1478 & 39 & 46 & 256 & 410 & 39 & 39 \\
\hline \multirow{8}{*}{785} & 20 & 23 & 46 & 39 & 448 & 19 & 23 & 23 & 23 \\
\hline & 40 & 33 & 61 & 134 & 2015 & 28 & 33 & 33 & 187 \\
\hline & 80 & 33 & 71 & 150 & 3164 & 28 & 33 & 33 & 1056 \\
\hline & 100 & 33 & 71 & 150 & 3164 & 28 & 33 & 33 & 1056 \\
\hline & 120 & 33 & 71 & 150 & 3164 & 28 & 33 & 33 & 1056 \\
\hline & 160 & 33 & 71 & 150 & 3164 & 28 & 33 & 33 & 1056 \\
\hline & 180 & 33 & 71 & 150 & 3164 & 28 & 33 & 33 & 1056 \\
\hline & 200 & 28 & 61 & 150 & 2999 & 23 & 33 & 28 & 983 \\
\hline
\end{tabular}

As shown in Fig. 6(a), there is the grating absorbance of four different periods for the changeable thickness at $\lambda=785 \mathrm{~nm}$. For the matching grating period $570 \mathrm{~nm}(n=1)$ of this wavelength, the absorbance is about 0.16 when the thickness is larger than $50 \mathrm{~nm}$. We can conclude that if the thickness is large enough, the absorbance will not change anymore. Because when the Ag film is too thick, the reflection becomes dominant. And the absorbance changes rapidly when the thickness is less than $50 \mathrm{~nm}$. Because the $\mathrm{Ag}$ film is too thin, the photon absorbance will be less probable for the small interaction distance. So an optimal range of Ag thickness must exist for which the probability of photon reflection and transmission are appropriate, and the probability of photon absorbance is high enough. As shown in Fig. 6(b), when the grating period is $570 \mathrm{~nm}$, which is close to the wavelength of the excitation light wavelength, two absorbance 
peaks exist at $\lambda=442 \mathrm{~nm}$ and $\lambda=802 \mathrm{~nm}$, respectively. For a fixed wavelength, the absorbance of the matching grating period with $n=1$ is larger

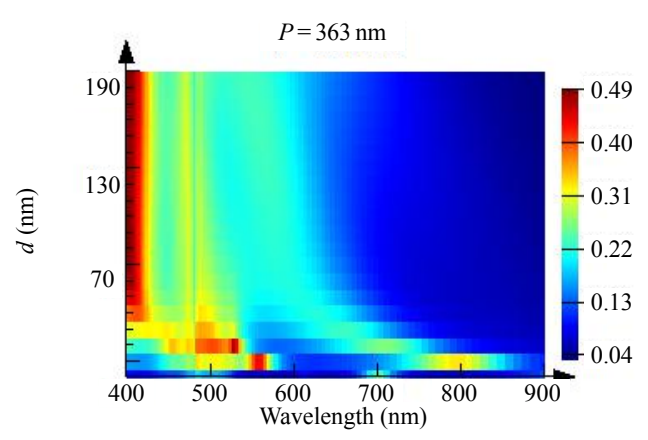

(a)

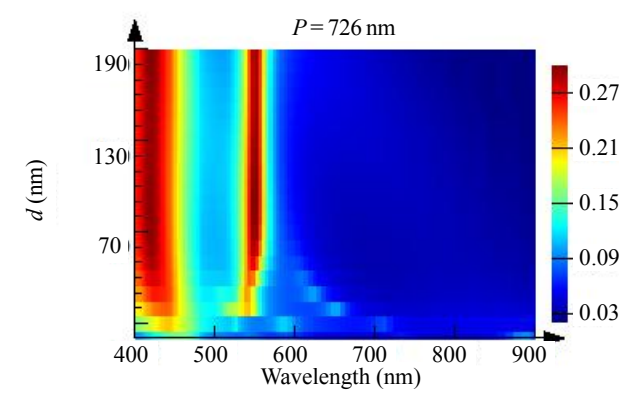

(c) than other $n$ other cases. Thus, the grating period plays a very significant role in the SPPs excitation.

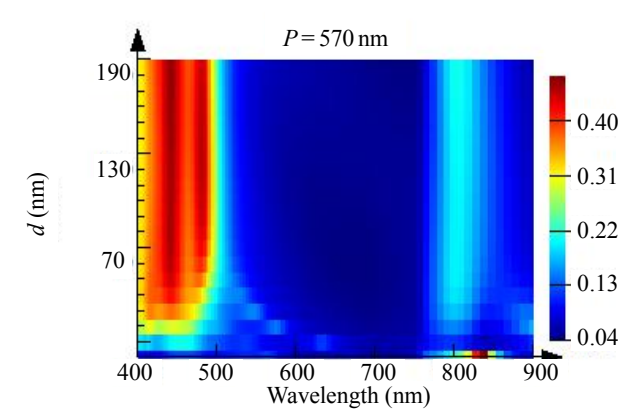

(b)

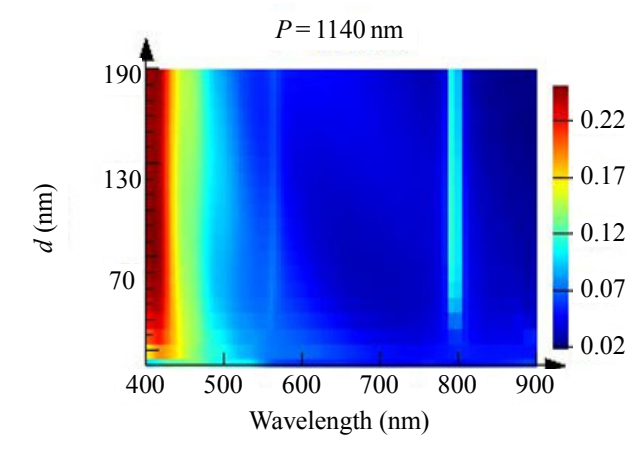

(d)

Fig. 5 Calculated absorbance as the function of the excitation light wavelength and Ag layer thickness for different periods: (a)-(d) correspond to $P=363 \mathrm{~nm}, 570 \mathrm{~nm}, 726 \mathrm{~nm}$, and $1140 \mathrm{~nm}$, respectively.

\subsection{Amplitude of the 1D sinusoidal grating}

The grating amplitude is another parameter that can affect the SERS response. To take this parameter into account, the maximum of absorbance value dependent on the Ag thickness and excitation light wavelength at different amplitudes with the constant period $P=570 \mathrm{~nm}$ and $\lambda=785 \mathrm{~nm}$ is performed as shown in Fig. 6(c). The maximum of photon absorbance is relatively bigger at the amplitude $A=15 \mathrm{~nm}$. From Table 2, we can see that the maximum EF also exists at $A=15 \mathrm{~nm}$. Besides, the absorbance remains about the same when the thickness of the Ag layer is greater than $75 \mathrm{~nm}$. From Fig. 6(d), it is clearly that the absorbance peak exists at $\lambda=802 \mathrm{~nm}$. In addition, the position of the absorbance peak is slightly effected by the variation of the amplitude, especially near the absorbance peak at $\lambda=451 \mathrm{~nm}(n=2)$. In Table 2 , we can see that the SERS EF is related to the amplitude of the grating. By controlling the grating amplitude, the EF can be increased to 5 orders of magnitude when $\lambda=785 \mathrm{~nm}$. Hence, for the optimal SERS response, the effect of the grating amplitude should be taken into consideration. Hence, with the excitation wavelength $785 \mathrm{~nm}$, we can obtain the optimal SERS substrate based on the 1D sinusoidal nanograting with the nanogratong period $P=570 \mathrm{~nm}, \mathrm{Ag}$ layer thickness $d=100 \mathrm{~nm}$, and nanograting amplitude $A=15 \mathrm{~nm}$. 


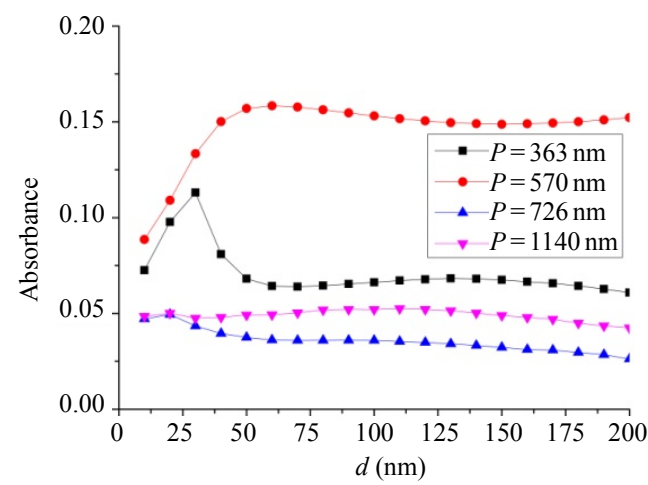

(a)

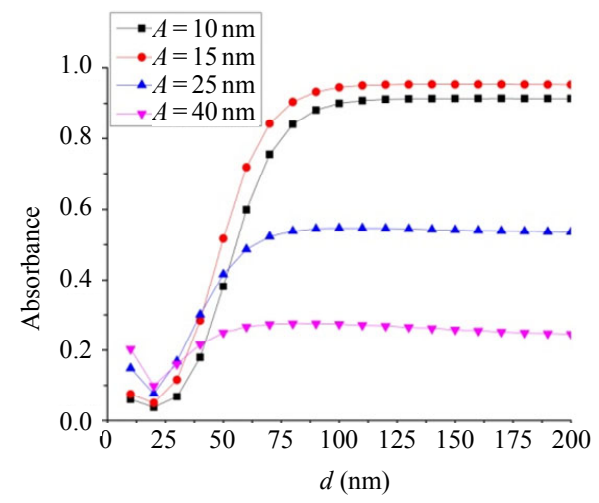

(c)

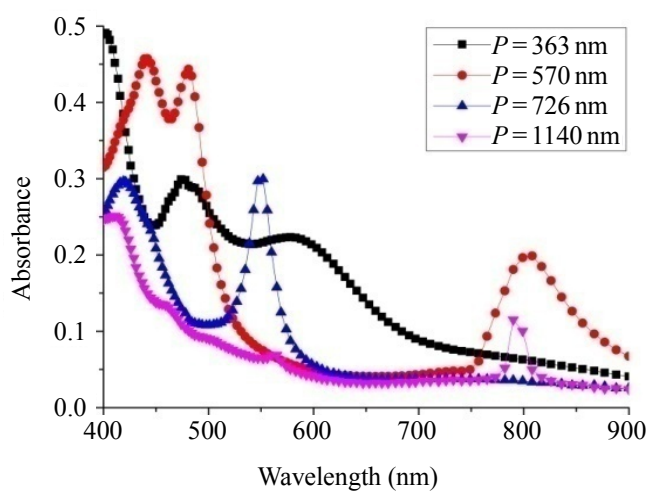

(b)

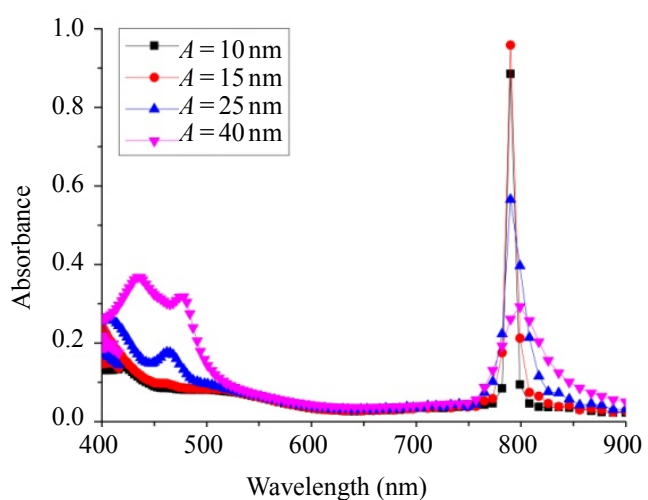

(d)

Fig. 6 Analysis of the period and amplitude of the 1D sinusoidal Ag nanograting: (a) calculated absorbance as the function of the Ag layer thickness for different periods at $\lambda=785 \mathrm{~nm}$, (b) calculated absorbance as the function of the excitation light wavelength for different periods at $d=100 \mathrm{~nm}$, (c) calculated absorbance as the function of the Ag layer thickness for different amplitudes at $\lambda=$ $785 \mathrm{~nm}$, and (d) calculated absorbance as the function of the excitation light wavelength for different amplitudes at $d=100 \mathrm{~nm}$.

Table $2 \mathrm{EF}$ as a function of the grating amplitude at $d=$ $100 \mathrm{~nm}$.

\begin{tabular}{cc}
\hline Amplitude $(\mathrm{nm})$ & EF \\
\hline 10 & 101124 \\
15 & 119025 \\
20 & 63001 \\
25 & 30976 \\
40 & 6724 \\
50 & 3164 \\
\hline
\end{tabular}

\section{Long-range SERS performance of the optimal 1D sinusoidal nanograting}

The electric field distribution simulations of the surface region of the optimal $1 \mathrm{D}$ sinusoidal $\mathrm{Ag}$ nanograting are implemented by the FDTD method. The electric field distribution of the $x z$ section is shown in Fig. 7(a), and the polarization angle of excitation light is $0^{\circ}$ [the zero polarization direction is parallel with $+x$ axis in Fig. 4(a)]. So EF can be $10^{5}$. What's more, in actual trace explosives detection, the distance size $L$ between the grating surface and the detected explosive molecules is also random, which plays an important role in SERS EF. As shown in Fig. 7(b), EF can be as high as $10^{4}-10^{5}$ when $L$ is small than $120 \mathrm{~nm}$. It demonstrates that a relatively big effective SERS detection range exists above the 1D sinusoidal nanograting. It indicates that the $1 \mathrm{D}$ sinusoidal nanograting has a long-range SERS effect. Compared with the traditional SERS substrate, such as the rough surface, nanospheres, nanopillar, the hotspots areas of these nanostructures SERS substrates are relatively small, and a short-range SERS effect exists in these substrates. On account of this long-range SERS effect, we can deduce that this SERS substrate is suitable for gas 
analysis tasks with no need of label molecules, such as SERS detection of trace explosives.

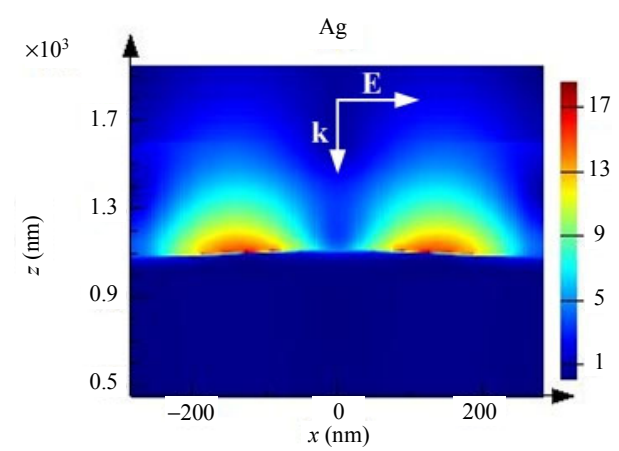

(a)

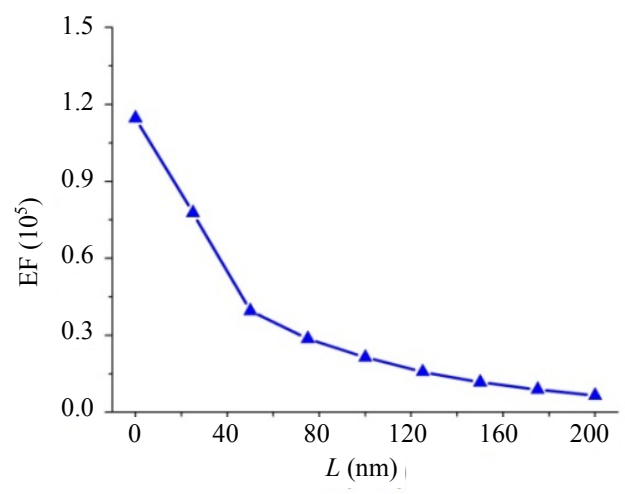

(b)

Fig. 7 Long-range SERS performance analysis of the 1D sinusoidal Ag nanograting: (a) electric field distribution of the $x z$ section and (b) EF vs the distance size between the grating surface and the detected molecules.

\section{Experiments and discussion}

For the sinusoidal Ag nanograting, the dielectric part of grating could be fabricated by the process of two-beam laser interference of the photoresist, and the Ag layer could be thermally evaporated onto the dielectric part. The photoresist (PR) (NOA-63) was spin coated onto a glass substrate pre-cleaned by an ultrasonic processing in alcohol, acetone, piranha acid, and deionized (DI) water in sequence. The continuous wave laser (Coherent MBD-266) with the wavelength of $266 \mathrm{~nm}$, power of $30 \mathrm{~mW}$, which was expanded by a lens array, was divided into two beams by a splitter to interfere on the PR layer. The interference period was controlled by the angle of two beams, for $P=\lambda / 2 \sin (\theta / 2)$, where $P$ is the grating period, $\lambda$ is the interference laser wavelength, and $\theta$ is the angle between two beams, as shown in Fig. 8(a). For example, when the optimal grating period is $570 \mathrm{~nm}, \theta$ should be $27^{\circ}$. The grating depth (double amplitude of sinusoidal grating) could be adjusted by changing the exposure time with a photoelectric shutter. The thickness of Ag layer could be adjusted by controlling the evaporation time (evaporation rate $0.1 \mathrm{~nm} / \mathrm{s}$ ). In the experiment, the optimal 1D sinusoidal nanograting was prepared, and its morphology was characterized with the scanning electron microscopy (SEM), as shown in Fig. 8(b). This structure of the sinusoidal Ag grating could be integrated onto the bottom of microchannel for the efficient label-free SERS detection of trace TNT, as shown in Fig. 9(c).

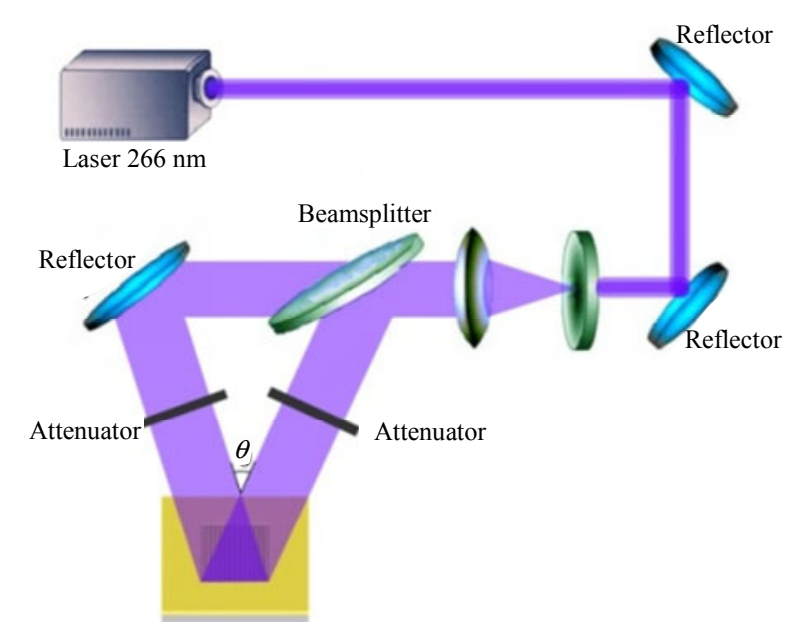

(a)

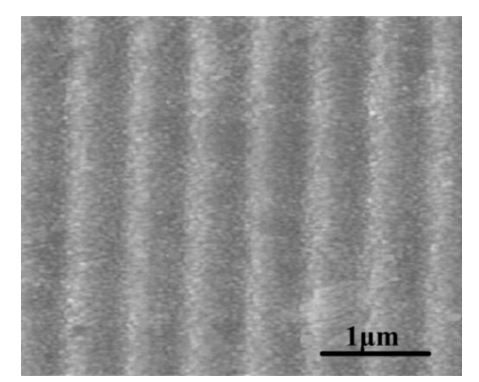

(b)

Fig. 8 Preparation of the 1D sinusoidal Ag nanograting: (a) schematic illustration of two-beam interference fabrication of the 1D sinusoidal nanograting and (b) the scanning electron microscopy (SEM) image of the 1D sinusoidal Ag nanograting.

In order to access SERS performance of the 1D sinusoidal Ag nanograting, we used common 
Rhodamine 6G (Rh6G) as a probe. The SERS signal was measured with a Raman probe (Inphotonics RPB fiber optic Raman probe) and a Raman spectrometer (Ocean Optics USB4000). The excitation laser was supported with Ocean Optics laser-785. Five Raman spectra for average values were measured at different positions on the 1D sinusoidal nanograting, and the tested Rh6G

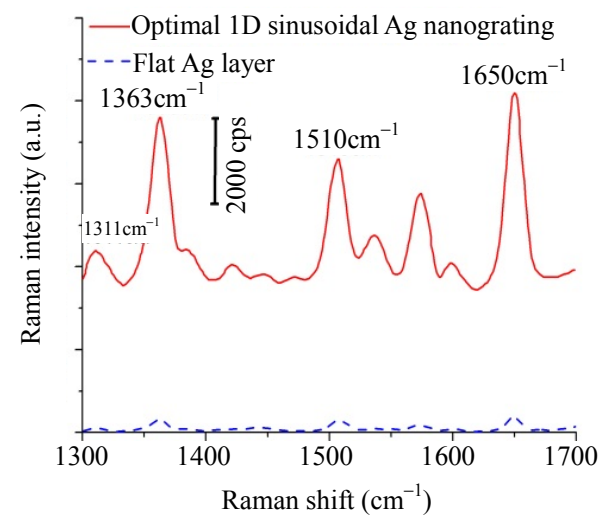

(a)

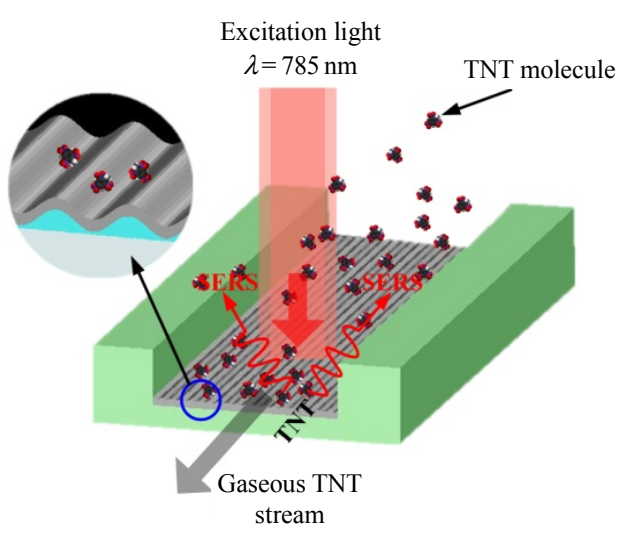

(c) concentration was $10^{-5} \mathrm{M}$. The results were shown in Fig. 9(a). The intensity of Rh6G Raman signal at $1650 \mathrm{~cm}^{-1}$ is 4316 . The mean values and standard deviations of the relative Raman intensity at four peaks are shown in Fig.9(b). The relative standard deviations of the relative Raman intensity at $1311 \mathrm{~cm}^{-1}, 1363 \mathrm{~cm}^{-1}, 1510 \mathrm{~cm}^{-1}$, and $1650 \mathrm{~cm}^{-1}$ are $5.8 \%, 13.4 \%, 9.4 \%$, and $11.8 \%$, respectively.

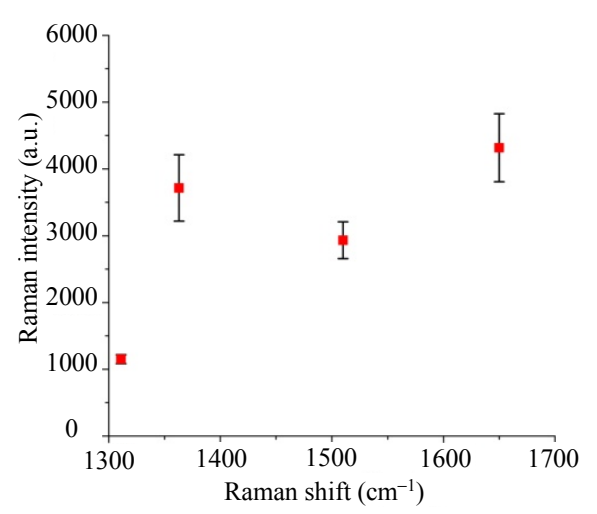

(b)

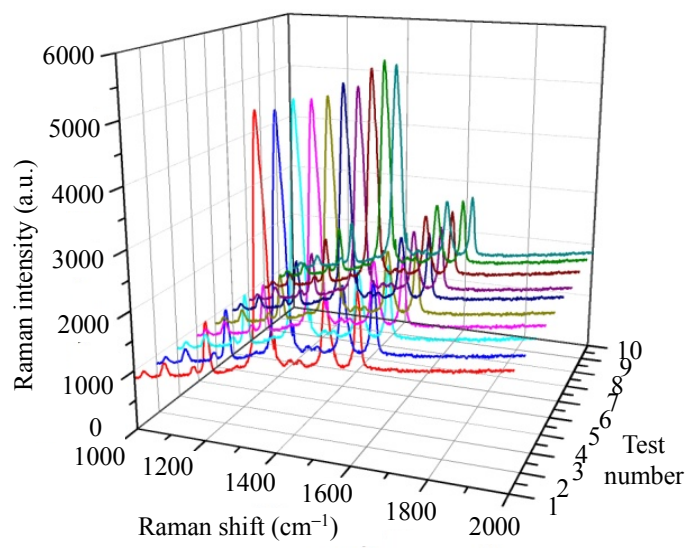

(d)

Fig. 9 SERS performance experiments of the 1D sinusoidal Ag nanograting: (a) Rh6G Raman spectra from the flat Ag layer and 1D sinusoidal nanograting, (b) error bars of the relative Raman intensities from the 1D sinusoidal nanograting, (c) schematic illustration of the label-free SERS detection of trace TNT, and (d) ten times detection of trace TNT with 5 minutes interval.

An analytical enhancement factor (AEF) is presented to evaluate the SERS EF from the optimal 1D sinusoidal nanograting as follows:

$$
A E F=\frac{I_{\mathrm{SERS}} / C_{\mathrm{SERS}}}{I_{\mathrm{RS}} / C_{\mathrm{RS}}}
$$

where $I_{\text {SERS }}$ and $C_{\text {SERS }}$ are the Raman signal intensity and the Rh6G concentration from the SERS substrate, respectively. $I_{\mathrm{RS}}$ and $C_{\mathrm{RS}}$ are the Raman signal intensity and the Rh6G concentration from the non-SERS substrate, respectively. In Fig. 9(a), the Rh6G concentration on the flat Ag layer is $10^{-2} \mathrm{M}$, and the corresponding intensity of Rh6G Raman signal at $1650 \mathrm{~cm}^{-1}$ is 45 . So the AEF of the optimal SERS substrate is about $9.6 \times 10^{4}$, and this measured EF value is approximately by order of 
magnitude lower than the calculated $\mathrm{EF}$ of the optimal SERS substrate. This disagreement is probably due to some differences between the ideal and real structures. The results prove that the optical enhancement observed on the Ag nanograting surface is mainly due to the plasmons coupling, which could be tuned by variation of periodic topologies. Hence, an optimized SERS enhancement is excepted when the period of the Ag nanograting is tuned to match with the excitation source in SERS experiments.

The experiment of the label-free SERS detection of trace TNT was carried out, and the SERS detection schematic illustration is shown in Fig. 9(c). The TNT solution was kept flowing above the surface of the 1D sinusoidal nanograting, and the TNT concentration was $10^{-5} \mathrm{M}$. Ten times TNT detection experiments with 5 minutes interval were conducted. The TNT Raman spectra are shown in Fig. 9(d), and we can see the TNT Raman spectra intensities are almost the same. So we can summarize that the SERS substrate based on the 1D sinusoidal nanograting has a good Raman signal reproducibility and can realize the label-free SERS detection of trace TNT. Although the SERS EF of the $1 \mathrm{D}$ sinusoidal nanograting is about $10^{4}$, it is much less than that for Raman signals obtained from TNT molecules absorbed on the SERS substrate. The result implies that it is impossible to concentrate TNT only in the enhanced electric field range above the surface of the 1D sinusoidal nanograting. This leads to averaging of Raman signals over the volume from which the scattered radiation is collected. Despite this fact, there are grounds to believe that the optimization of SPPs surface parameters can lead to an increase in the electric field near this surface, so that the SERS EF of this substrate can be improved, and the TNT detection sensitivity will be significantly increased.

\section{Conclusions}

In this paper, we show a composite $1 \mathrm{D} \mathrm{Ag}$ sinusoidal nanograting aiming at the label-free SERS detection of trace TNT. Theoretical and experimental investigations into excitation SPPs on the 1D sinusoidal Ag nanograting are performed with the aim to make SERS response as powerful as possible. The result shows that a long-range SERS effect exists in this substrate, which can overcome the memory effect and the noncontinuous work drawbacks of the traditional SERS substrates. Its label-free SERS detection capacity of gaseous trace TNT is demonstrated in the experiments. This SERS substrate provides a novel idea for the further real-time, label-free testing, and monitoring of trace agents.

\section{Acknowledgment}

This work was supported by the National Defense Science Technology Project Fund (Grant No. 2004053).

Open Access This article is distributed under the terms of the Creative Commons Attribution 4.0 International License (http://creativecommons.org/licenses/by/4.0/), which permits unrestricted use, distribution, and reproduction in any medium, provided you give appropriate credit to the original author(s) and the source, provide a link to the Creative Commons license, and indicate if changes were made.

\section{References}

[1] C. Wang and C. X. Yu, "Analytical characterization using surface enhanced Raman scattering (SERS) and microfluidic sampling," Nanotechnology, 2015, 26(9): $1-26$.

[2] Q. L. Li, B. W. Li, and Y. Q. Wang, "Surface-enhanced Raman scattering microfluidic sensor," Rsc Advances, 2013, 3(32): 13015-13026.

[3] C. Farcau and S. Astilean, "Periodically nanostructured substrates for surface enhanced Raman spectroscopy," Journal of Molecular Structure, 2014, 1073(1073): 102-111.

[4] E. L. Holthoff, D. N. Stratis-Cullum, and M. E. Hankus, "A nanosensor for TNT detection based on molecularly imprinted polymers and surface enhanced Raman scattering," Sensors, 2011, 11(3): 2700-2714.

[5] S. Chang, H. Ko, S. Singamaneni, R. Gunawidjaja, and V. V. Tsukruk, "Nanoporous membranes with mixed nanoclusters for Raman-based label-free 
monitoring of peroxide compounds," Analytical Chemistry, 2014, 81(14): 5740-5748.

[6] C. Li, C. L. Wu, J. S. Zheng, J. P. Lai, C. L. Zhang, Y. B. Zhao, et al., "LSPR sensing of molecular biothiols based on noncoupled gold nanorods," Langmuir the Acs Journal of Surfaces \& Colloids, 2010, 26(11): 9130-9135.

[7] D. E. Charles, D. Aheme, M. Gara, D. M. Ledwith, Y. K. Gunko, J. M. Kelly, et al., "Versatile solution phase triangular silver nanoplates for highly sensitive plasmon resonance sensing," Acs Nano, 2010, 4(1): 55-64.

[8] M. S. Goh, Y. H. Lee, S. Pedireddy, I. Y. Phang, W. W. Tjiu, J. M. Rui, et al., "A chemical route to increase hot spots on silver nanowires for surface-enhanced Raman spectroscopy application," Langmuir the Acs Journal of Surfaces \& Colloids, 2012, 28(40): 1444-1-1444-9.

[9] Y. F. Fang, X. L. Cheng, C. Y. Zhang, and Y. Zhou, "Review on graphene based explosive sensors," Chinese Journal of Energetic Materials, 2014, 22(1): 116-123.

[10] D. V. Petrov, A. R. Zaripov, and N. A. Toropov, "Enhancement of Raman scattering of a gaseous medium near the surface of a silver holographic grating," Optics Letters, 2017, 42(22): 4728-4731.

[11] L. Chen and J. Choo, "Recent advances in surface-enhanced Raman scattering detection technology for microfluidic chips," Electrophoresis, 2008, 29(9): 1815-1828.

[12] P. B. Johnson and R. W. Christy, "Optical constants of the noble metals," Physical Review B, 1972, 6(12): 4370-4379.

[13] J. Tang, H. Guo, M. Chen, J. T Yang, and D. Tsoukalas, "Wrinkled Ag nanostructured gratings towards single molecule detection by ultrahigh surface Raman scattering enhancement," Sensors \& Actuators B Chemical, 2015, 218: 145-151.

[14] M. J. Banholzer, J. E. Millstone, L. Qin, and C. A. Mirkin, "Rationally designed nanostructures for surface-enhanced Raman spectroscopy," Chemical Society Reviews, 2008, 37(5): 885-897.

[15] H. F. Ghaemi, T. Thio, D. E. Grupp, T. W. Ebbesen, and H. J. Lezec, "Surface plasmons enhance optical transmission through subwavelength holes," Physical Review B, 1998, 58(11): 6779-6782.

[16] R. Gillibert, M. Sarkar, J. F. Bryche, J. Moreau, M. Besbes, G. Barbillon, et al., "Directional surface enhanced Raman scattering on gold nano-gratings," Nanotechnology, 2016, 27(11): 115202-1-115202-9.

[17] T. W. Lee and S. K. Gray, "Subwavelength light bending by metal slit structures," Optics Express, 2005, 13(24): 9652-9659.

[18] A. Taflove and S. C. Hagness, Computational electrodynamics: the finite difference time domain method. Boston, USA: Artech House, 2005: 1-839.

[19] Y. Kalachyova, D. Mares, O. Lyutakov, M. Kostejn, L. Lapcak, and V. Svorcik, "Surface plasmon polaritons on silver gratings for optimal SERS response," Journal of Physical Chemistry C, 2015, 119(17): 9506-9512. 